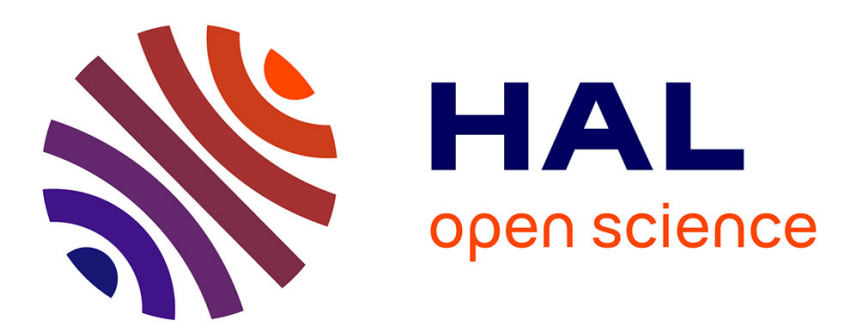

\title{
Simulation en laboratoire de l'attachement d'un leader à une structure flottante
}

\author{
G. Labaune, F. Issac, J.-P. Moreau, A. Bondiou, J.-C. Alliot, B. Hutzler, F. \\ Morillon
}

\section{> To cite this version:}

G. Labaune, F. Issac, J.-P. Moreau, A. Bondiou, J.-C. Alliot, et al.. Simulation en laboratoire de l'attachement d'un leader à une structure flottante. Revue de Physique Appliquée, 1990, 25 (2), pp.131-137. 10.1051/rphysap:01990002502013100 • jpa-00246169

\section{HAL Id: jpa-00246169 https://hal.science/jpa-00246169}

Submitted on 1 Jan 1990

HAL is a multi-disciplinary open access archive for the deposit and dissemination of scientific research documents, whether they are published or not. The documents may come from teaching and research institutions in France or abroad, or from public or private research centers.
L'archive ouverte pluridisciplinaire HAL, est destinée au dépôt et à la diffusion de documents scientifiques de niveau recherche, publiés ou non, émanant des établissements d'enseignement et de recherche français ou étrangers, des laboratoires publics ou privés. 


\title{
REVUE DE PHYSIQUE APPLIQUÉE
}

Revue Phys. Appl. 25 (1990) 131-137

FÉVRIER 1990, PAGE 131

Classification

Physics Abstracts

$92.90-93.85-52.90$

\section{Simulation en laboratoire de l'attachement d'un leader à une structure flottante}

\author{
G. Labaune $\left({ }^{1}\right)$, F. Issac $\left({ }^{1}\right)$, J.-P. Moreau $\left({ }^{1}\right)$, A. Bondiou $\left({ }^{1}\right)$, J.-C. Alliot $\left({ }^{1}\right)$, B. Hutzler $\left({ }^{2}\right)$ et \\ F. Morillon (2) \\ ( $\left.{ }^{1}\right)$ Office National d'Etudes et de Recherches Aérospatiales, BP 72, 92322 Châtillon Cedex, France \\ (2) EDF Centre des Renardières, Moret sur Loing, France
}

(Reçu le 27 février 1989, accepté le 19 septembre 1989)

\begin{abstract}
Résumé. - La connexion entre un éclair atmosphérique et un avion est étudiée grâce à des expériences en vol. Malheureusement, celles-ci sont mal adaptées à l'étude de la formation des décharges électriques à l'extérieur de l'appareil c'est pourquoi des expériences de laboratoire ont été entreprises pour étudier la formation et le développement des canaux de décharge lors de leur connexion avec des structures électriquement flottantes.

Deux expériences de laboratoire présentées ici permettent de préciser la phénoménologie de la connexion et montrent en particulier que les signaux enregistrés sur avion sont caractéristiques d'un amorçage de la décharge à partir de la structure.
\end{abstract}

\begin{abstract}
The connection between an atmospheric lightning and an aircraft is studied by the way of inflight experiments. Unfortunately, these ones are not matched to the study of electrical discharges in the surroundings of the aircraft. For this reason laboratory experiments have been carried out to study the connection between long sparks and electrically floating structures.

Two laboratory experiments give results concerning the physical process involved in the connection and show that data recorded during inflight experiments are representative of an ignition of the discharge on the aircraft itself.
\end{abstract}

\section{Introduction.}

Le problème qui nous concerne est celui de la protection des équipements embarqués sur avion lors d'impact direct de foudre. Il est apparu lors d'expériences en vol que la phase de jonction éclairavion se caractérise par une séquence d'impulsions de champs électrique et magnétique et de courant à la surface de l'appareil. Malheureusement, les expériences embarquées sont mal adaptées à l'étude des mécanismes intervenant dans la physique du développement des décharges. A titre d'exemple, l'analyse d'enregistrements vidéo du développement des décharges ne permet pas de définir si la décharge est initiée par l'avion ou si, lors de sa propagation, elle vient toucher l'avion. Une description électrique et radioélectrique de ces décharges est toutefois nécessaire, car, pour une protection correcte des avions, il est nécessaire de définir des termes de source (courants ou champs), qui appliqués en tout point de l'avion, à l'aide de codes numériques, permettront de calculer le «cas majorant " en un point donné de la structure.

C'est pourquoi, depuis plusieurs années, nous avons entrepris des expériences de laboratoire dont le but est l'étude de la connexion, par des décharges électriques, d'objets électriquement flottants.

Rappel des principaux résultats obtenus lors d'expériences passées.

Une première expérience [1] a été effectuée en 1985 pour étudier la connexion entre un cylindre métallique électriquement flottant et des décharges électriques de quelques dizaines de centimètres de longueur. Il a été montré que les variations rapides de 
champ électrique de surface apparaissent à la surface $\mathrm{du}$ cylindre pendant la phase de connexion, et que celles-ci sont dues aux variations brutales de résistance des canaux de décharge.

Plusieurs études relatives aux décharges électriques à la pression atmosphérique parmi lesquelles nous pouvons citer les décharges de surface $[2,3]$, les décharges dans les courts intervalles d'air [4], et les décharges dans les longs intervalles d'air [5], ont conduit à deux conclusions importantes.

La première de ces conclusions est que le milieu, dans son évolution vers l'état arc, passe toujours par les transitions classiques de la physique des décharges à haute pression [6] que sont l'apparition de l'effet de charge d'espace et le chauffage du milieu par les collisions coulombiennes. La première conduit à l'état streamer qui est caractérisé macroscopiquement par un champ électrique longitudinal dans le canal qui ne dépend que de la polarité de la décharge. Le second permet le chauffage des neutres et la chute du champ électrique, conduisant à l'état leader.

La seconde de ces conclusions est que, si l'on considère un système constitué de deux électrodes entre lesquelles on applique une tension suffisante pour obtenir le claquage, on doit distinguer deux cas. Dans le premier, la chute de résistance du milieu se produit après que le canal de la décharge ait traversé tout l'intervalle. C'est le cas d'une "décharge courte »; l'évolution du courant est alors fonction du circuit extérieur et de la variation de résistance du canal. Dans le second, la chute de résistance du milieu se produit pendant la propagation de la décharge dans l'intervalle.

Pendant la propagation de cette « décharge longue », constituée d'un streamer et d'un leader, l'évolution du courant n'est fonction que des propriétés physiques de la décharge elle-même. Les expériences de laboratoire sur ce type de décharges ont montré que ces propriétés sont très différentes pour les décharges des deux polarités : courant quasiment continu dans le cas de la propagation d'une décharge positive et courant impulsionnel associé à la propagation par bonds de la décharge négative.

L'expérience citée plus haut, utilisant des claquages dans des intervalles de quelques dizaines de centimètres, est représentative des propriétés des « décharges courtes ». L'éclair atmosphérique appartient de manière évidente à la catégorie des « décharges longues ». Aussi, les considérations précédentes nous ont-elles amenés en 1986 à entreprendre une expérience pour étudier la connexion du même cylindre métallique par des décharges de quelques mètres de longueur.

Le cylindre (longueur $4 \mathrm{~m}$, diamètre $0,5 \mathrm{~m}$ ) a été placé dans l'intervalle pointe-plan de $14 \mathrm{~m}$ disponible sous le générateur $6 \mathrm{MV}$ du centre EDF des Renardières. Nous ne parlerons ici que des résultats obtenus dans le cas des essais en polarité négative de la pointe. L'expérience a montré que la propagation par bonds de la décharge dans l'intervalle inférieur induisait des impulsions de champs électrique et magnétique à la surface du cylindre [1].

La figure 1 représente le dispositif expérimental. La figure 2a montre les enregistrements simultanés de la tension à la pointe, des champs électrique et magnétique à la surface du cylindre et d'un caméragramme obtenu à l'aide du convertisseur d'image en mode balayage. Ce caméragramme permet de visualiser l'évolution temporelle de la décharge dans les deux intervalles. La figure $2 \mathrm{~b}$ représente un grossissement du caméragramme et de l'enregistrement du champ électrique de surface au moment de l'amorçage de la décharge négative se propageant dans l'intervalle inférieur.

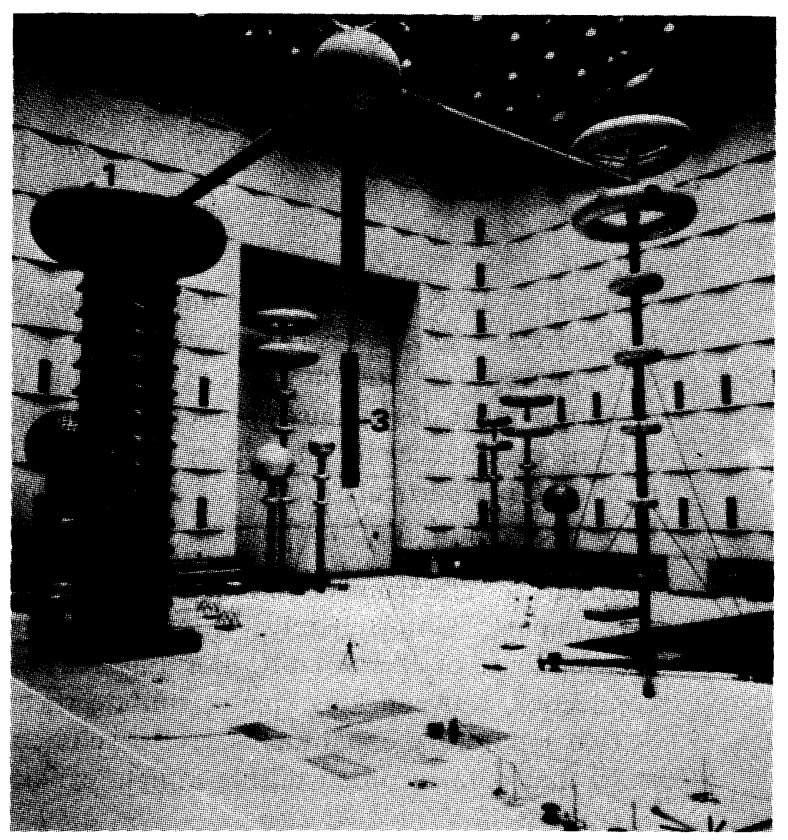

Fig. 1. - Dispositif expérimental de l'expérience en configuration pointe-plan : 1) générateur haute tension; 2) diviseur capacitif de mesure de la tension sphère ; 3) cylindre électriquement flottant.

[Experimental set-up : rod-plane configuration.]

La séquence des phénomènes intervenant dans la connexion peut être décrite de la manière suivante :

Lorsque la tension à la pointe atteint une valeur suffisante, des streamers négatifs apparaissent à la base de celle-ci. Ils ont la structure d'un buisson de filaments. Ils sont associés à une impulsion de courant à la pointe atteignant une valeur crête de quelques centaines d'ampères.

Un système streamer-leader négatif se propage en direction de la partie supérieure du cylindre et on remarque qu'aucune variation importante de champ électrique ou magnétique n'est observée à la surface de la structure pendant cette propagation. 

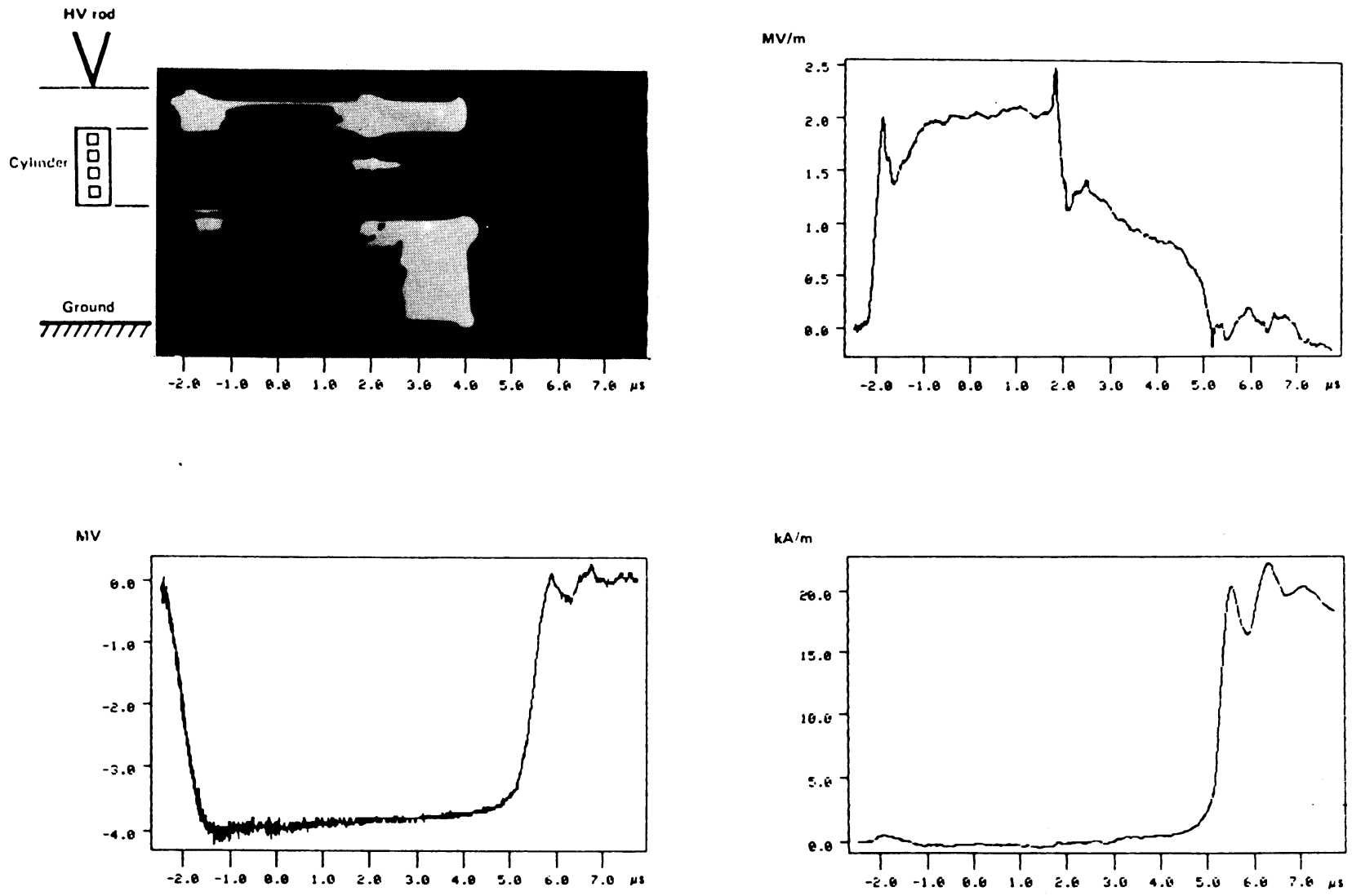

a)
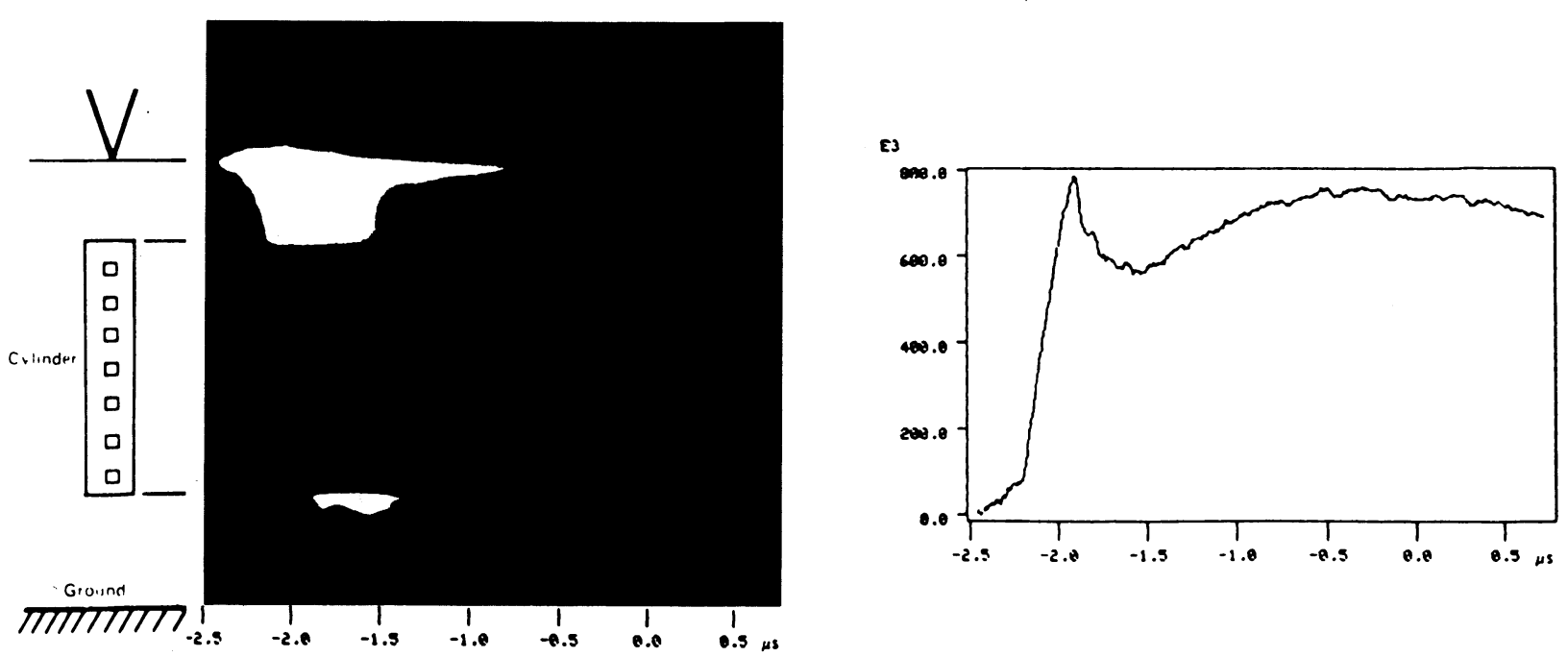

b)

Fig. 2. - Résultats expérimentaux en configuration pointe-plan. (a) Vue générale de la connexion. (b) Vue de détail : amorçage du streamer négatif dans l'intervalle inférieur.

[Experimental results : rod plane configuration : (a) general view of the connection ; (b) negative streamer ignition in the lower gap.] 
Lorsque la jonction est effectuée dans l'intervalle supérieur, le champ électrique à la surface du cylindre s'accroît rapidement, corrélativement avec la montée en potentiel du cylindre qui se charge à travers la résistance que constitue l'intervalle supérieur.

Cette montée en potentiel induit l'apparition, à la base du cylindre, d'une décharge négative. Celle-ci s'éteint rapidement car on remarque (Fig. 2b), que le courant nécessaire à la formation de cette décharge provoque, en circulant dans la forte résistance de l'intervalle supérieur, une chute de potentiel du cylindre. L'extinction de cette décharge permet la remontée en potentiel de la structure (donc du champ électrique à sa surface), avec une constante de temps $R C, R$ étant la résistance équivalente à l'intervalle supérieur, et $C$ la capacité de la structure.

Pendant les 3 microsecondes qui suivent, rien n'apparaît sur aucun des enregistrements. Après quoi, une variation de la conductivité dans l'intervalle supérieur provoque la montée en potentiel de la structure qui induit elle-même le réamorçage de la décharge dans l'intervalle inférieur.

Le même scénario que précédemment se produit, avec chute de potentiel du cylindre, extinction de la décharge, début de recroissance du potentiel, puis l'intervalle étant franchi, la jonction s'effectue avec le sol ou avec des streamers positifs venant du sol. On remarque que pendant la phase suivante, le cylindre est en équilibre électrique entre les deux résistances que constituent les deux intervalles, et que ces résistances sont encore de forte valeur puisqu'un courant très faible circule dans le circuit ainsi fermé. Ce n'est qu'au temps $T_{0}$ qu'une évolution rapide de la résistance du milieu permettra l'établissement du fort courant de décharge.

Cette expérience conduit à plusieurs remarques :

Les valeurs maximales des dérivées temporelles des champs électrique et magnétique de surface sont comparables à celles enregistrées sur avion. Les valeurs de dérivées que nous obtenons ici étant liées aux variations de résistance du milieu et aux impulsions de courant associées aux amorçages des décharges, nous pouvons émettre l'hypothèse selon laquelle les mécanismes physiques élémentaires mis en jeu sont les mêmes que dans le cas de la connexion avion.

Malheureusement, l'allure générale de l'évolution temporelle du champ électrique de surface n'est pas du tout représentative de celle observée sur avion; en particulier elle ne rend pas compte de la lente variation du champ électrique qui est toujours observée sur avion au début du processus de connexion (Fig. 3).

Nous avons supposé que cette différence venait du fait que l'initiation de la décharge se produisait sur l'avion dans le cas réel contrairement à ce qui se produit dans la configuration expérimentale pointe-

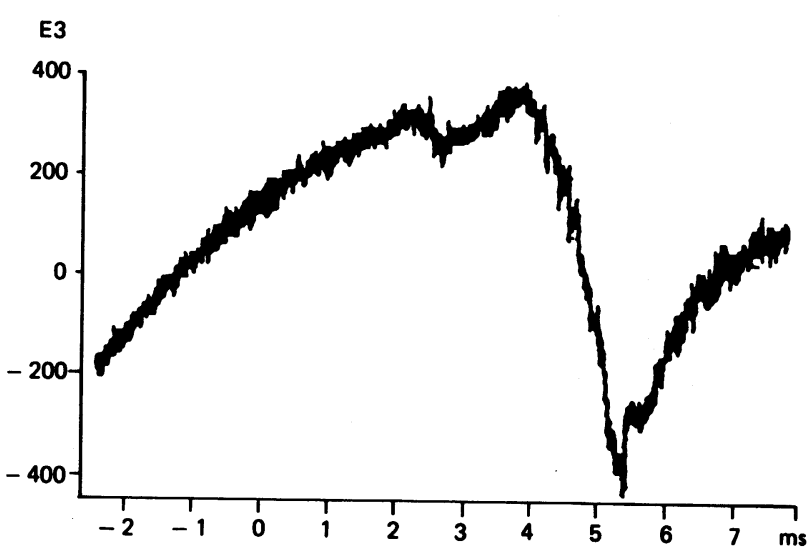

Champ électrique de surface

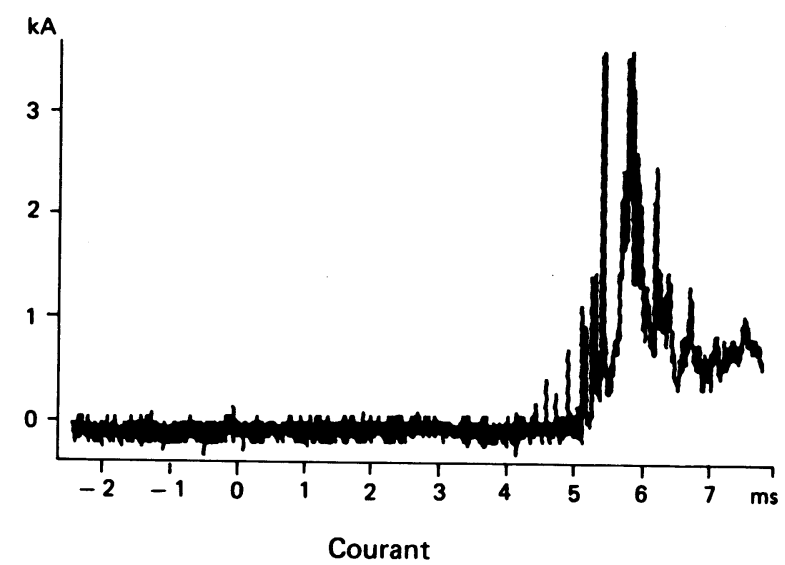

Fig. 3. - Variations simultanées du champ électrique de surface et du courant traversant l'avion pendant la connexion éclair-avion.

[Simultaneous temporal evolution of the surface electric field and of the current flowing through the aircraft during its connection with the lightning.]

plan où la décharge, initiée à la pointe haute tension se propage jusqu'à la structure.

Aussi avons nous réalisé en 1987, une expérience utilisant le même générateur, mais dans laquelle la structure est placée dans un intervalle plan-plan.

\section{Expérience en configuration plan-plan.}

DISPOSITIF EXPÉRIMENTAL ET DIAGNOSTICS. - La figure 4 représente le dispositif expérimental. Une maquette d'avion (avion Transall à l'échelle 1/10) est suspendue par des fils diélectriques dans l'intervalle plan-plan formé par le sol et un disque de diamètre $5,6 \mathrm{~m}$.

Le générateur haute tension est le même que dans l'expérience précédente.

Les diagnostics utilisés sont les suivants :

- des convertisseurs d'image électronique, fonctionnant en mode balayage de fente ou image intégrale dans les domaines visible et ultraviolet; 


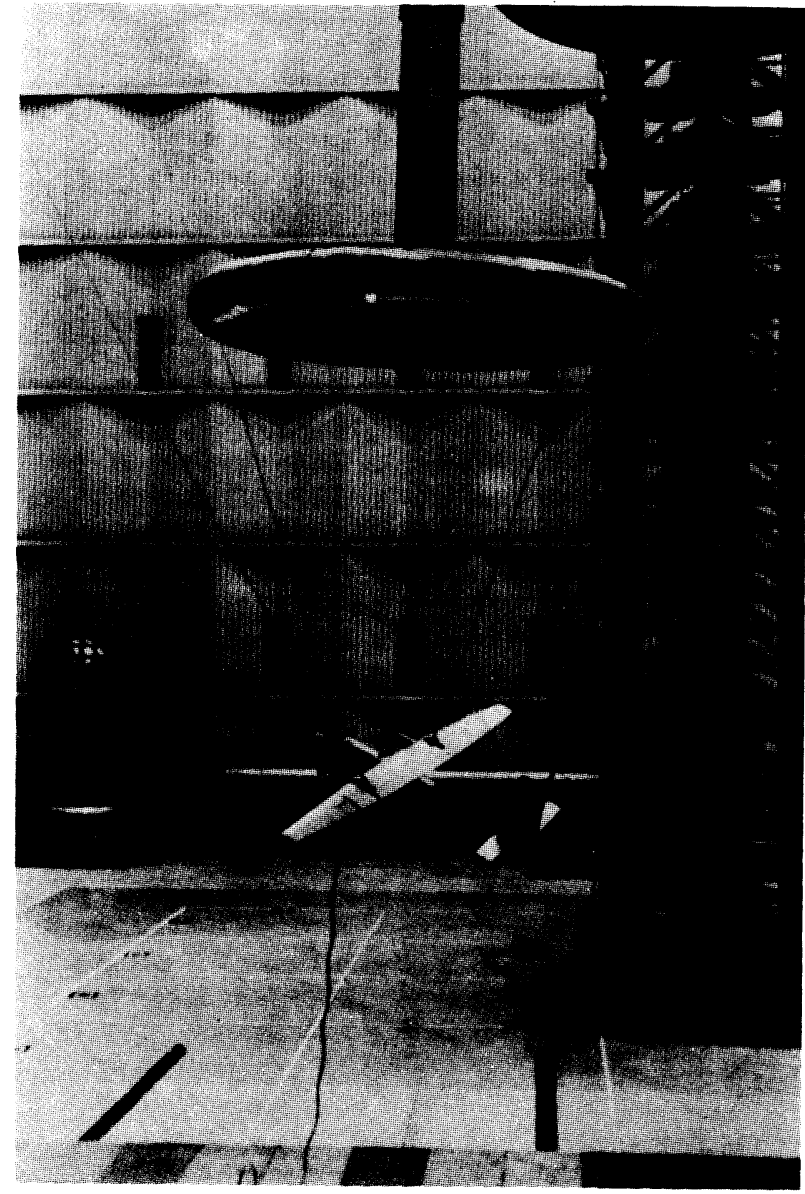

Fig. 4. - Dispositif expérimental de l'expérience en configuration plan-plan.

[Experimental set-up : plane-plane configuration.]

- un diviseur capacitif mesure la tension du plateau avec une bande passante de $3 \mathrm{MHz}$;

- des capteurs $\mathrm{d} i / \mathrm{d} t$ sont intégrés dans les perches placées à l'avant, à l'arrière et aux extrémités des ailes de la maquette (Fig. 5a) ;

- des capteurs $\mathrm{d} E / \mathrm{d} t$ et $\mathrm{d} H / \mathrm{d} t$ de surface sont placés sur les ailes et sur le fuselage de la maquette. La figure $5 b$ représente ces capteurs sur l'aile de la maquette ;

- tous les signaux sont transmis par des fibres optiques de bande passante $150 \mathrm{MHz}$ et numérisés au moyen de Tektronix RTD 710.

\section{Résultats.}

La figure 6 montre une image obtenue à l'aide d'une caméra vidéo pendant la connexion déchargemaquette. Elle permet de connaître, pour chaque tir, les points d'entrée et de sortie de la décharge sur la maquette. Le cas présenté ici représente une connexion nez-queue. D'autres positions de la maquette permettraient d'obtenir d'autres modes de

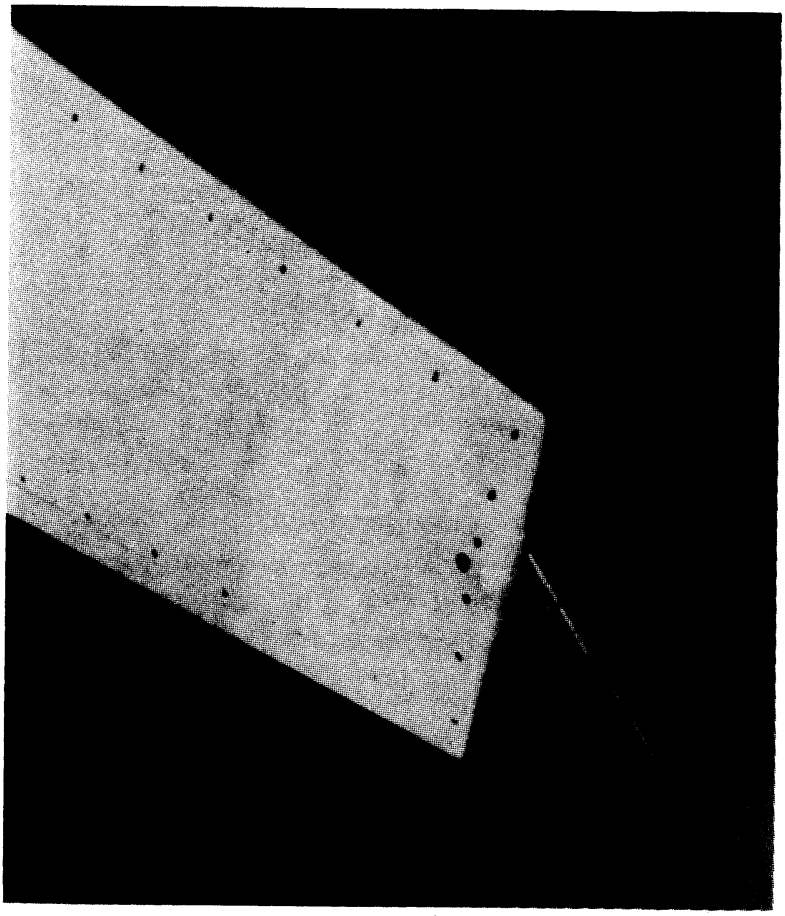

a)

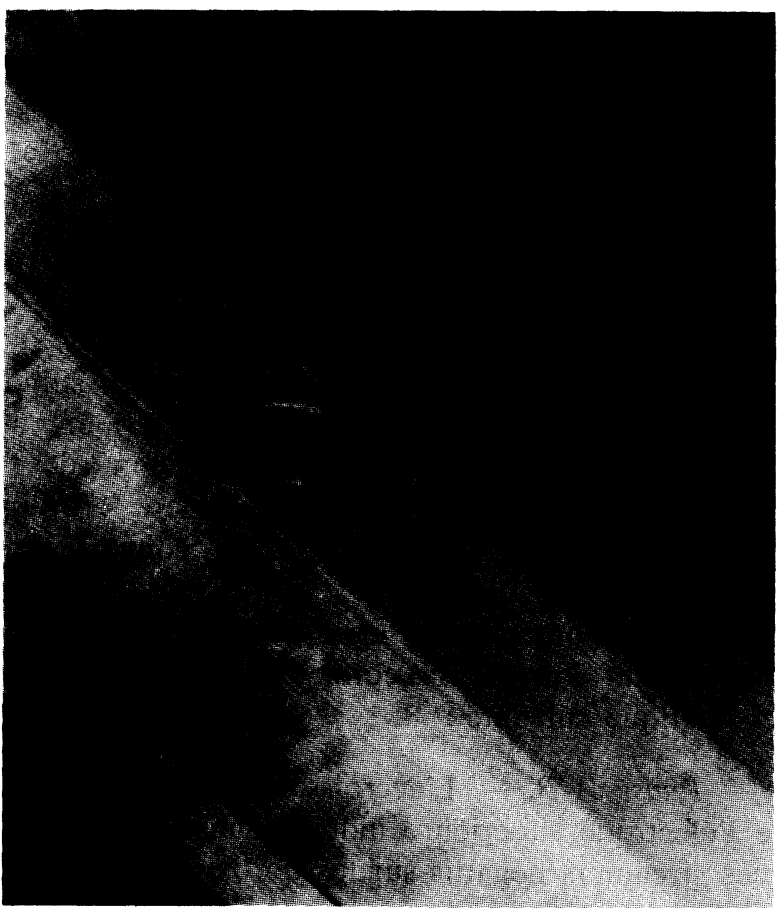

b)

Fig. 5. - Capteurs équipant la maquette d'avion Transall. (a) Capteur $\mathrm{d} i / \mathrm{d} t$ d'extrémité d'aile. (b) Capteur $\mathrm{d} E / \mathrm{d} t$ et $\mathrm{d} H / \mathrm{d} t$ d'aile.

[Sensors of the Transall aircraft mock-up. (a) $\mathrm{d} i / \mathrm{d} t$ sensor on the wing ; (b) $\mathrm{d} E / \mathrm{d} t$ and $\mathrm{d} H / \mathrm{d} t$ on the wing.]

connexion (nez-aile...). Il est à noter que, pour une position donnée de la maquette, le mode de connexion est très reproductible. 


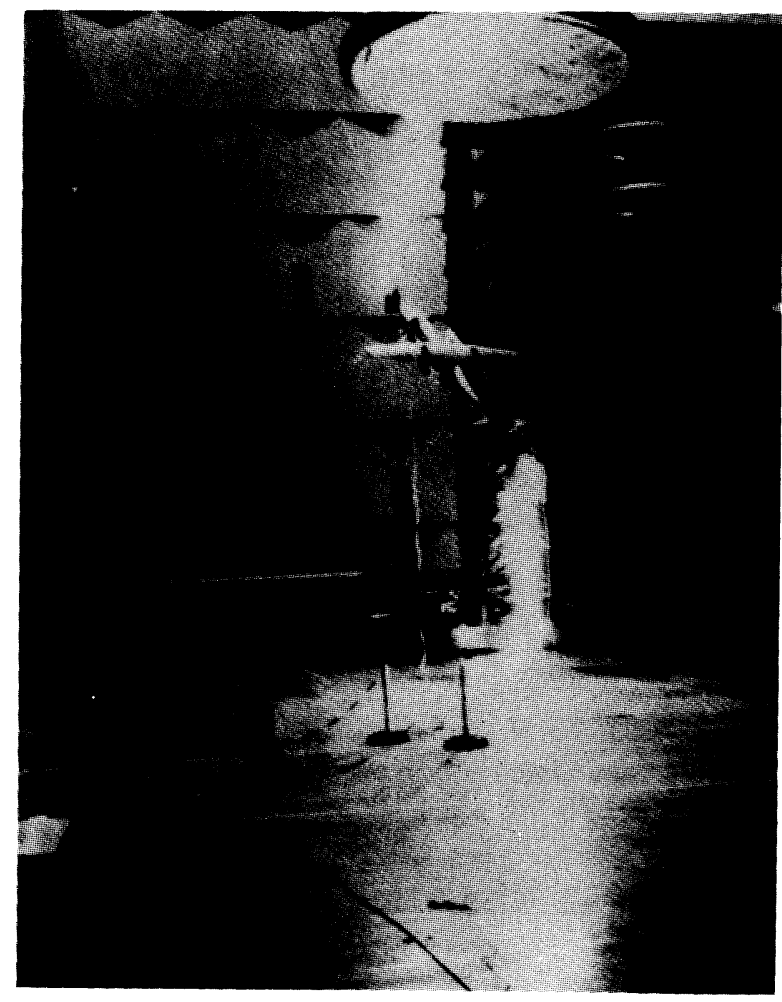

Fig. 6. - Enregistrement vidéo d'une connexion nezqueue.

[Video recording of the nose-tail connection.]

La figure 7 représente des enregistrements simultanés de la tension sur le plateau supérieur, du champ électrique à la surface de l'avion, de la dérivée du courant à la perche arrière et d'un caméragramme obtenu à l'aide du convertisseur d'image en mode balayage.

Nous pouvons décrire les événements successifs apparaissant lors de la connexion de la manière schématique suivante :

- Phase A-B. - L'accroissement de la tension de l'électrode supérieure induit l'émission de quelques streamers négatifs sur les bords du disque. La taille de ces streamers n'est pas suffisante pour atteindre la maquette et ceux-ci ne semblent avoir aucune influence sur les événements suivants.

En ce qui concerne les signaux enregistrés sur la maquette, on voit que l'accroissement de la tension de l'électrode supérieure provoque une augmentation du champ électrique par effet d'influence.

- Phase B-C. - Le caméragramme montre, dans l'intervalle supérieur, le développement d'un système streamer-leader positif et dans l'intervalle inférieur, l'apparition de quelques streamers négatifs.

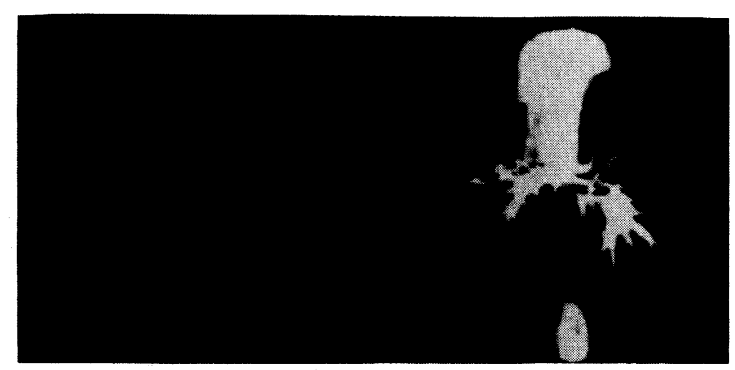

a)

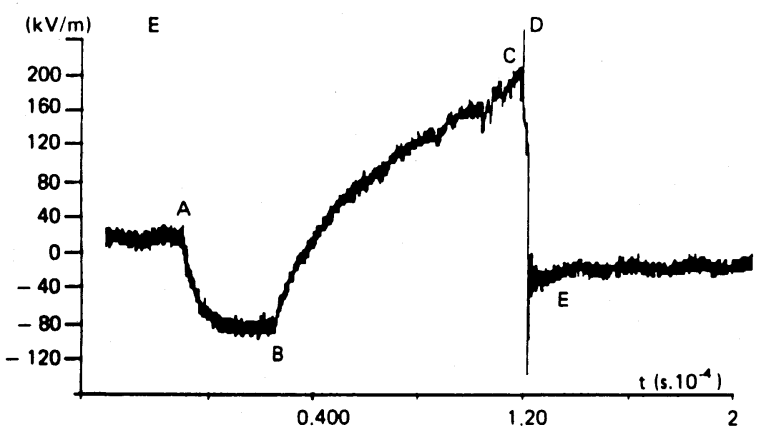

b)

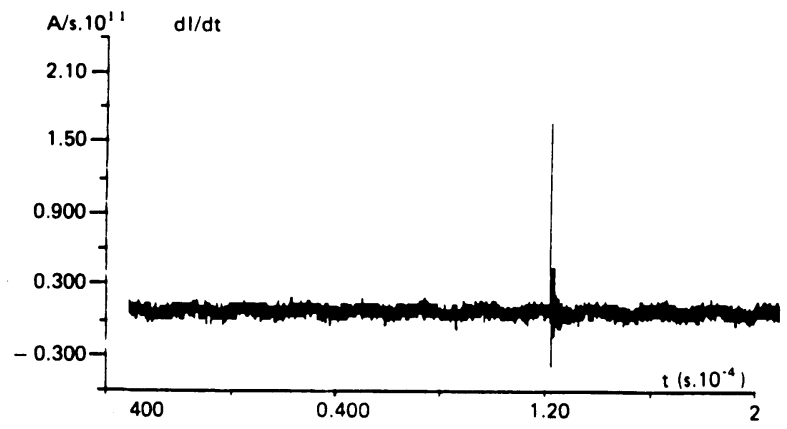

c)

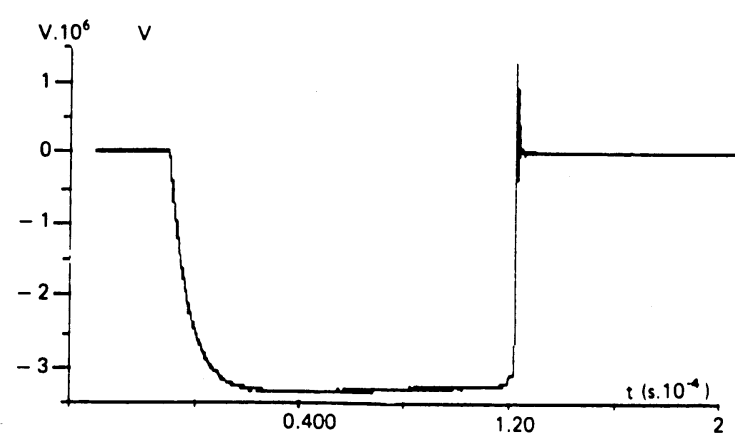

d)

Fig. 7. - Résultats expérimentaux dans le cas de la configuration plan-plan. (a) Caméragramme obtenu en mode balayage. (b) Champ électrique enregistré à l'avant du fuselage. (c) Dérivée du courant mesuré sur la perche avant. (d) Tension sur le plateau.

[Data recorded in the plane-plane configuration. (a) Streak cameragram. (b) Surface electric field on the fuselage. (c) Current derivative on the nose boom. (d) Upper plane voltage.] 
L'effet global de ces deux phénomènes sur le champ électrique de surface, se traduit par une augmentation représentative de la variation du potentiel de la structure.

On doit noter que les streamers positifs se développant dans l'intervalle supérieur franchissent très rapidement celui-ci pour atteindre l'électrode haute tension. Ainsi, le système maquette-décharge n'est plus, à proprement parler, électriquement flottant. Nous discuterons plus loin la limitation apportée à l'expérience par ce phénomène.

Les streamers négatifs se développent dans l'intervalle inférieur de manière impulsionnelle et sont de plus en plus importants à mesure que le potentiel de la structure devient plus négatif : près du point $B$, ils sont très faibles et l'on peut considérer qu'à cet instant le potentiel de la structure ne varie qu'en fonction de sa capacité et du courant nécessaire à la formation du système streamer-leader positif de l'intervalle supérieur ; vers le point $\mathrm{C}$, les streamers négatifs plus importants provoquent, lors de leur apparition, des variations décelables du potentiel de la structure.

- Phase $C-D$. - A partir du point $\mathrm{C}$, le processus est le même que celui décrit dans le cadre de l'expérience précédente (configuration pointe-plan). Une variation rapide de la résistance du canal dans l'intervalle supérieur provoque une augmentation du potentiel de la structure.

- Phase D-E. - Un leader négatif se développe alors par bonds dans l'intervalle inférieur, atteint le sol et le courant peut s'écouler dans le circuit ainsi fermé.

\section{Conclusion et discussion.}

Les valeurs crête des dérivées temporelles des champs électrique et magnétique de surface sont respectivement $1,5 \times 10^{13} \mathrm{~V} / \mathrm{m} / \mathrm{s}$ et $1 \times 10^{11} \mathrm{~A} / \mathrm{m} / \mathrm{s}$. Ces grandeurs sont tout à fait comparables à celles obtenues lors de l'expérience en configuration pointe-plan et comparables également à celles obtenues lors des expériences en vol $\left(1,5\right.$ à $5 \times 10^{12} \mathrm{~V} / \mathrm{m} / \mathrm{s}$ et $6 \times 10^{9}$ à $2 \times 10^{10} \mathrm{~A} / \mathrm{m} / \mathrm{s}$ [8]).

D'autre part, la forme générale de l'évolution du champ électrique de surface, qui, dans l'expérience précédente n'était pas satisfaisante devient correcte. On peut donc considérer que notre supposition était fondée, et admettre comme une hypothèse raisonnable que la phase de lente variation du champ électrique observée sur avion au début du processus de connexion est associée au développement d'un leader positif émanant de l'avion lui-même.

Le fait que, dans notre dispositif expérimental, le système streamer-leader positif se développant dans l'intervalle supérieur fasse très rapidement une connexion avec l'électrode haute tension est une limitation. En effet, il interdit l'étude du démarrage du leader négatif par bonds dans la phase où il doit se développer en équilibre électrique avec le leader positif, comme ce processus se produit dans le cas de l'avion.

Pour analyser ces phénomènes importants pour la compréhension de la connexion éclair-avion, de nouvelles expériences doivent être menées, conjointement par EDF et l'ONERA, utilisant des intervalles de claquage plus grands et des champs électriques plus uniformes.

\section{Bibliographie}

[1] Levesque P., Taillet J., Labaune G., Larigaldie S., Alliot J. C., A study of the physical mechanisms and the perturbations created by the attachment of an arc to a conducting cylinder, 10th ICOLSE, Paris (juin 1985).

[2] LARIGALDIE S., The spark propagation mechanisms in ambiant air at the surface of a charged dielectric, I. Experimental : the main stages of the discharge, J. Appl. Phys. 61 (1) 1987a.

[3] LARIGALDIE S., The spark propagation mechanisms in ambiant air at the surface of a charged dielectric, II. Theoretical modelling, J. Appl. Phys. 61 (1) 1987b.
[4] Mesyats G. A. and Korshunov G. S., Sov. Phys. Tech. Phys. $13 \mathrm{n}^{\circ} 4$ (1968).

[5] Renardières Group, Electra, 74, 1981.

[6] TAILLET J., Basic phenomenology of electrical discharges at atmospheric pressure, AGARD Lecture Series $\mathrm{n}^{\circ} 110$.

[7] LABAUNE G. et al., Experimental study of the interaction between an arc and electrically floating structure, 11th ICOLSE, Dayton (June 1986).

[8] Moreau J. P. and Alliot J. C., Analysis of the first milliseconds of aircraft lightning attachment, 11th ICOLSE, Dayton (June 1986). 